\title{
Plasma n-3 and n-6 fatty acids and inflammatory markers in Chinese vegetarians
}

\author{
Xiaomei Yu ${ }^{1 *}$, Tao Huang ${ }^{2}$, Xiumei Weng ${ }^{1}$, Tianxing Shou ${ }^{3}$, Qiang Wang ${ }^{1}$, Xiaogiong Zhou' ${ }^{1}$, Qinxin Hu ${ }^{1}$ \\ and Duo Li ${ }^{3 *}$
}

\begin{abstract}
Background: Polyunsaturated fatty acid (PUFA) intake favorably affects chronic inflammatory-related diseases such as cardiovascular disease; however, the relationship between the PUFA and inflammatory factors in the healthy vegetarians were not clear. We aimed to investigate the plasma fatty acids status, and its association with plasma inflammatory factors in Chinese vegetarians and omnivores.

Methods: A total of 89 male vegetarians and 106 male omnivores were participated the study. Plasma concentrations of inflammatory factors were detected by ELISA, and as standard methods fatty acids were extracted and determined by chromatography.

Results: Compared with omnivores, vegetarians have significant higher interleukin-6 (IL-6), plasma n-6 PUFA, n-6/n-3, and 18:3n-3; while they have significant lower leukotriene B4 (LTB4), cyclo-oxygenase-2 (COX2) and prostaglandin E2 (PGE2), 20:5n-3, 22:5n-3, 22:6n-3, and n-3 PUFA. In vegetarians, plasma 20:4n-6 was significant positively related to TNF-a. LTB4 was significantly positively related to plasma 22:6n-3, and negatively associated with n-6 PUFA.

Conclusion: Vegetarians have higher plasma n-6 PUFA and IL-6, but lower LTB4, n-3 PUFA, 22:6n-3, COX2 and PGE2 levels. It would seem appropriate for vegetarians to increase their dietary n-3 PUFA, while reduce dietary $\mathrm{n}-6$ PUFA and thus reduce the risk of chronic inflammatory-related diseases.
\end{abstract}

Keywords: Polyunsaturated fatty acid, Vegetarians, Omnivores, Inflammatory factor

\section{Introduction}

It is widely recognized that overall mortality, ischemic heart disease mortality and overall cancer incidence are lower in vegetarians compared with omnivores [1,2]. The dietary patterns of vegetarians as well as their healthful lifestyle practices are thought to at least partly explain these differences. Vegetarian diets are rich in fiber, magnesium, $\mathrm{Fe}^{3+}$, folic acid, vitamins $\mathrm{C}$ and $\mathrm{E}, \mathrm{n}-6$ polyunsaturated fatty acid (PUFA), phytochemicals, and antioxidants [3]. Another notable difference relates to the type and amount of fat in the diet. Vegetarian diets are slightly lower in total fat than omnivorous diets $[4,5]$. Low intake of total fat, saturated fatty acids (SFA), and sodium and high intake of fiber, phytochemicals, and antioxidants in

\footnotetext{
*Correspondence: yuxiaomei_1@hotmail.com; duoli@zju.edu.cn 'Department of Clinical Laboratory, Zhejiang Hospital, 12 Linyin Road, Hangzhou 310030, China

${ }^{3}$ Department of Food Science and Nutrition, Zhejiang University, 866 Yuhangtang Road, Hangzhou 310059, China

Full list of author information is available at the end of the article
}

vegetarians is associated with low blood pressure and body mass index [3].

However, vegetarians diets are low in sodium, zinc, $\mathrm{Fe}^{2+}$, vitamins $A$, vitamins $B_{12}$, and $D$, and $n-3$ polyunsaturated fatty acid (PUFA) [3,6]. With respect to intake of essential fatty acid, especially n-3 PUFA, vegetarian diets appear to offer no advantages over omnivorous dietary patterns. It has been suggested that vegetarians could be at a significant disadvantage, as consumption of $\alpha$-linolenic acid $(18: 3 n-3)$ islow, resulting in limited conversion of $18: 3 n-3$ to eicosapentaenoic acid (20:5n-3) and docosahexaenoic acid (22:6n-3) [7]. In addition, ovolacto vegetarian could get limited amount of 20:5n-3 and 22:6n-3, however, vegan diet almost has no $20: 5 n-3$ and $22: 6 n-3$, they may get tiny amount from algae and seaweed [4].

Eicosanoids, which are the mediators and regulators of inflammation, are generated from 20-carbon PUFA. Because inflammatory cells typically contain a high proportion of the n-6 PUFA arachidonic acid (20:4n-6) and low 
proportions of other 20-carbon PUFA [8], 20:4n-6 is a substrate of 2- and 4-series eicosanoids, which are associated with inflammation. Many anti-inflammatory pharmacotherapies are directed at inhibiting the production of these inflammatory mediators and thus possibilities exist for therapies that incorporate n-3 PUFA [9]. High level of n-3 PUFA in the diet can suppress the production of both tumor necrosis factor $\alpha$ (TNF- $\alpha)$ and interleukin $1 \beta$ (IL-1 $\beta)$ [10]. Dietary supplementation with encapsulated fish oil rich in 20:5n-3 and 22:6n-3 has been shown to result in decreased synthesis of TNF- $\alpha$ and interleukin-1 $\beta$ (IL-1 $\beta$ ) monocyte by in healthy subjects [10].

To date, no study reported the relationship of plasma fatty acids with blood inflammatory factors in Chinese vegetarians. Therefore, the purpose of the present study was to investigate the status of plasma fatty acids and to examine the potential relationship between fatty acids and plasma inflammatory factors in Chinese vegetarians and omnivores.

\section{Materials and methods Subjects}

The study protocol was approved by the Ethics Committee, Zhejiang Hospital, Hangzhou, China, and all subjects were volunteers who gave their written consent prior to participation in the study. A total of 89 male vegetarians (aged $35.39 \pm 12.2$ year) were recruited in Linyin Temple, Hangzhou, China. A vegetarian was defined as someone who ate no red meat, fish and chicken, and had been following this diet for at least 6 months prior to the study. A total of 106 male omnivores (aged $36.62 \pm 9.8$ year) were recruited through a health check program during the period of October 2010 through March 2011 in the Zhejiang Hospital, Hangzhou, China. An omnivore was defined as someone who ate meat at least five times per week.

\section{Blood collection}

Subjects attended the Zhejiang Hospital in the morning following an overnight fast. Subjects were allowed to sit relaxed for $10 \mathrm{~min}$, the subject's weight, height, waist to hip ratio and blood pressure were measured. Then venous blood was taken in plain and EDTA vacuum tubes with 21-gauge needles. After blood collection, plasma samples were prepared quickly after blood was drawn, aliquoted into separate tubes and stored at $-20^{\circ} \mathrm{C}$ until analysis.

\section{Laboratory measurements}

Total lipid of plasma was extracted with chloroform:methanol 1:1 (C:M, v/v) containing $10 \mathrm{mg} / \mathrm{L}$ of butylated hydroxytoluene (Tokyo Kasei Kogyo Co., Ltd. Japan). The plasma phospholipid (PL) fractions were separated by thin-layer chromatography. The methyl esters of the fatty acids of the plasma PL fractions were prepared using $0.9 \% \mathrm{H} 2 \mathrm{SO} 4$ in methanol containing $35 \mathrm{mg} / \mathrm{L}$ of n-Nonadecanoic acid (C19:0) (Chem Service, PA, USA) as internal standards. The fatty acid composition of plasma PL was determined by capillary gaseliquid chromatography using an Agilent $60 \mathrm{~m}-0.25 \mathrm{~mm}-0.25 \mathrm{~mm}$ column [11,12]. IL-6, LTB4, COX2, PGE2 were determined by ELISA kits (R\&D Systems, USA) through standard method in Clinical laboratory, Zhejiang Hospital, China.

\section{Statistical analysis}

Data analyses were performed using SAS for Windows, version 9.1 (SAS Institute). All continuous variables were examined for normal distribution. Differences between the two groups for each outcome were analyzed using t-test. The associations between plasma fatty acid composition and inflammatory factors were determined by partial correlation, controlling for potential confounding factors. All data are expressed as mean \pm SD. Differences between groups were considered to be statistically significant at $p<0.05$.

\section{Results}

Compared with omnivores, the weight was significantly lower in vegetarians $(\mathrm{p}<0.001)$. IL-6 $(\mathrm{p}<0.001)$ was significantly higher in vegetarians, while LTB4 $(\mathrm{p}<0.001)$, COX2 $(\mathrm{p}<0.001)$ and PGE2 $(\mathrm{p}<0.001)$ were significantly lower in vegetarians $(P=0.005)$ (Table 1$)$.

Table 1 The demographic and biochemical measurements in vegetarians and omnivores

\begin{tabular}{llll}
\hline & $\begin{array}{l}\text { Vegetarians } \\
\mathbf{n = 8 9}\end{array}$ & $\begin{array}{l}\text { Omnivores } \\
\mathbf{n}=106\end{array}$ & P value \\
\hline Age $($ year $)$ & $35.39 \pm 12.2$ & $36.62 \pm 9.8$ & 0.444 \\
Height $(\mathrm{m})$ & $1.68 \pm 0.06$ & $1.72 \pm 0.05$ & $<0.001$ \\
Weight $(\mathrm{kg})$ & $66.96 \pm 10.52$ & $70.65 \pm 9.62$ & 0.012 \\
BMI $\left(\mathrm{kg} / \mathrm{m}^{2}\right)$ & $23.75 \pm 3.27$ & $23.76 \pm 2.80$ & 0.975 \\
Log IL-1 $(\mathrm{pg} / \mathrm{mL})$ & $0.11 \pm 0.029$ & $0.11 \pm 0.044$ & 0.430 \\
Log IL-2 $(\mathrm{pg} / \mathrm{mL})$ & $0.24 \pm 0.11$ & $0.27 \pm 0.13$ & 0.096 \\
Log IL-6 $(\mathrm{pg} / \mathrm{mL})$ & $2.33 \pm 0.11$ & $2.12 \pm 0.14$ & $<0.001$ \\
Log IL-10 $(\mathrm{pg} / \mathrm{mL})$ & $1.01 \pm 0.14$ & $1.03 \pm 0.21$ & 0.503 \\
Log TNF-a $(\mathrm{pg} / \mathrm{mL})$ & $0.17 \pm 0.058$ & $0.16 \pm 0.057$ & 0.536 \\
Log LTB4 $(\mathrm{pg} / \mathrm{mL})$ & $1.84 \pm 0.26$ & $1.98 \pm 0.20$ & $<0.001$ \\
Log COX2 $(\mathrm{mg} / \mathrm{mL})$ & $0.498 \pm 0.147$ & $0.610 \pm 0.173$ & $<0.001$ \\
Log PGE2 $(\mathrm{pg} / \mathrm{mL})$ & $1.82 \pm 0.150$ & $2.08 \pm 0.142$ & $<0.001$ \\
\hline
\end{tabular}

Data was expressed as Mean \pm SD.P $<0.05$ indicate the significant difference between groups.

t test was used to test the differences between groups.

TNF-a: tumor necrosis factor-alpha, IL: interleukin, LTB4: Leukotriene B4, COX2: cyclo-oxygenase 2, PGE2: prostaglandin E2. 
The plasma fatty acids composition was significantly different between vegetarians and omnivores. Compared with omnivores, the plasma 20:5n-3 ( $p<0.001), 22: 5 \mathrm{n}-3$ $(p<0.001), 22: 6 \mathrm{n}-3(p<0.001)$, and n-3 LC-PUFA $(p<$ $0.001)$ were significantly lower in vegetarians, while, the plasma n-6 PUFA $(p<0.001), \mathrm{n}-6 / \mathrm{n}-3 \quad(p<0.001)$, and 18:3n-3 $(p<0.001)$ were significantly higher in vegetarians (Table 2).

We used the partial correlations to test the associations between inflammatory factors and plasma fatty acids. In vegetarians, plasma 20:4n-6 $(\mathrm{r}=0.241, p<0.05)$ was significantly positively correlated with TNF- $\alpha$. LTB4 was significantly positively correlated with plasma $22: 6 \mathrm{n}$ $3(\mathrm{r}=0.241, p<0.05)$, and negatively correlated with $\mathrm{n}-6$ PUFA $(\mathrm{r}=-0.270, p<0.05)$ (Table 3$)$.

\section{Discussions}

In the present study, we found that vegetarians have higher plasma n-6 PUFA, n-6/n-3, 18:3n-3 and IL-6, lower plasma 20:5n-3, 22:5n-3, 22:6n-3, n-3 PUFA, LTB4, COX2 and PGE2.In vegetarians, plasma $20: 4 n-6$ was significantly positively correlated with TNF- $\alpha$. LTB4 was significantly positively related with plasma 22:6n-3.

Vegetarian diets are slightly lower in total fat than omnivorous diets [4]. Vegetarian diets are rich in n-6 PUFA, but, low in n-3 PUFA [3]. Ovolacto vegetarians consume

Table 2 Plasma fatty acid composition in vegetarians and omnivores

\begin{tabular}{|c|c|c|c|}
\hline \multirow{3}{*}{$\begin{array}{l}\text { PL Fatty acids } \\
\text { (\% of total } \\
\text { fatty acids) }\end{array}$} & \multicolumn{2}{|l|}{ Plasma $^{a}$} & \multirow{3}{*}{$\begin{array}{l}P \\
\text { value }\end{array}$} \\
\hline & Vegetarians & Omnivores & \\
\hline & $n=89$ & $n=106$ & \\
\hline $18: 2 n-6$ & $25.94 \pm 4.67$ & $24.08 \pm 3.12$ & 0.001 \\
\hline $18: 3 n-6$ & $0.34 \pm 0.20$ & $0.10 \pm 0.10$ & 0.000 \\
\hline $20: 2 n-6$ & $1.33 \pm 0.20$ & $0.44 \pm 0.24$ & 0.000 \\
\hline $20: 3 n-6$ & $4.99 \pm 2.51$ & $2.46 \pm 1.55$ & 0.000 \\
\hline $20: 4 n-6$ & $15.04 \pm 9.21$ & $10.87 \pm 2.73$ & 0.000 \\
\hline $22: 2 n-6$ & $0.49 \pm 0.45$ & $0.22 \pm 0.31$ & 0.000 \\
\hline $22: 4 n-6$ & $0.64 \pm 0.59$ & $0.39 \pm 0.27$ & 0.000 \\
\hline $18: 3 n-3$ & $0.39 \pm 0.29$ & $0.19 \pm 0.12$ & 0.000 \\
\hline $20: 5 n-3$ & $0.83 \pm 1.20$ & $1.34 \pm 1.13$ & 0.003 \\
\hline $22: 5 n-3$ & $1.09 \pm 0.42$ & $1.23 \pm 0.35$ & 0.020 \\
\hline $22: 6 n-3$ & $2.12 \pm 0.85$ & $4.66 \pm 1.45$ & 0.000 \\
\hline n-6 PUFA & $48.57 \pm 7.02$ & $38.84 \pm 3.05$ & 0.000 \\
\hline n-3 PUFA & $4.45 \pm 1.84$ & $7.41 \pm 2.07$ & 0.000 \\
\hline$n-6 / n-3$ & $12.33 \pm 4.70$ & $5.63 \pm 1.68$ & 0.000 \\
\hline
\end{tabular}

Data was expressed as Mean \pm SD.

${ }^{a}$ All fatty acids are significantly different between two groups $(p<0.05)$.

The difference between the vegetarians and omnivores is determined by using general linear model controlled potential confounding factors (Age and $\mathrm{BMI})$.

PUFA: polyunsaturated fatty acids, MUFA, monounsaturated fatty acids. O: omnivores, V: vegetarians, PL: phospholipids.
Table 3 Partial correlations between plasma fatty acid compositions and plasma inflammatory factors

\begin{tabular}{|c|c|c|c|c|c|c|c|}
\hline & & $20: 4 n-6$ & $20: 5 n-3$ & $22: 6 n-3$ & n-3 PUFA & n-6 PUFA & $n-6 / n-3$ \\
\hline \multirow[t]{2}{*}{ |L-1 } & V & 0.125 & -0.17 & -0.1 & -0.19 & 0.102 & -0.19 \\
\hline & $\mathrm{O}$ & 0.065 & 0.051 & 0.045 & 0.086 & -0.112 & 0.11 \\
\hline \multirow[t]{2}{*}{ IL-2 } & V & 0.098 & 0.127 & -0.13 & -0.06 & 0.039 & -0.06 \\
\hline & $\mathrm{O}$ & -0.063 & -0.093 & 0.016 & -0.04 & 0.007 & -0.039 \\
\hline \multirow[t]{2}{*}{ IL-6 } & V & -0.02 & -0.02 & -0.12 & -0.13 & -0.03 & -0.1 \\
\hline & $\mathrm{O}$ & 0.079 & 0.136 & 0.053 & 0.124 & -0.15 & 0.155 \\
\hline \multirow[t]{2}{*}{ IL-10 } & V & -0.14 & 0.037 & 0.041 & 0.083 & -0.2 & 0.132 \\
\hline & $\mathrm{O}$ & -0.112 & -0.154 & -0.047 & -0.171 & -0.042 & -0.15 \\
\hline \multirow[t]{2}{*}{ TNF-a } & V & $0.241^{*}$ & -0.09 & -0.13 & -0.14 & 0.207 & -0.19 \\
\hline & $\mathrm{O}$ & 0.071 & -0.008 & 0.061 & 0.059 & -0.096 & 0.08 \\
\hline \multirow[t]{2}{*}{ LTB4 } & V & -0.08 & -0.03 & $0.316^{*}$ & 0.077 & $-0.27^{*}$ & 0.136 \\
\hline & $\mathrm{O}$ & 0.005 & 0.08 & -0.122 & -0.096 & -0.14 & -0.056 \\
\hline \multirow[t]{2}{*}{ PGE2 } & V & 0.106 & -0.07 & 0.054 & 0.086 & -0.04 & 0.08 \\
\hline & $\mathrm{O}$ & 0.163 & 0.064 & 0.049 & 0.063 & 0.097 & 0.035 \\
\hline \multirow[t]{2}{*}{$\mathrm{COX} 2$} & V & -0.11 & -0.07 & 0.081 & 0.024 & -0.17 & 0.079 \\
\hline & $\mathrm{O}$ & -0.045 & 0.123 & 0.113 & 0.116 & -0.201 & 0.156 \\
\hline
\end{tabular}

The associations of plasma PL fatty acids compositions with plasma inflammatory factors centration was tested by using pearson partial correlation model after controlling confounding factor(age, BMI).

Coef, partial correlation coefficient, O: omnivores, V: vegetarians,

PL: phospholipids. ${ }^{*} \mathrm{p}<0.05$.

minimal amounts of 20:5n-3 and varying amounts of 22:6n-3 from eggs, milk, and dairy products. Vegans consume negligible amounts of n-3 PUFA and rely entirely on in vivo biosynthesis of $n-3$ PUFA from the precursor $18: 3 n-3$, but conversion via desaturation and elongation, especially to $22: 6 n-3$, is not efficient [12]. Previous study has shown conversion of $18: 3 n-3$ to $20: 5 n-3$ varying from $6-21 \%$ to much lower values(0.1-0.2\%), or undetectable 22:6n-3 synthesis [13]. In the present study, we found that vegetarians have higher plasma PL n-6 PUFA, n-6/n-3, and 18:3n-3, while lower plasma PL 20:5n-3, 22:5n-3, 22:6n-3, and n-3 long-chain (LC)-PUFA. Lack of 20:5n-3 and $22: 6 \mathrm{n}-3$ in vegetarian diets is reflected in reduced amounts of these fatty acids in platelets, $\mathrm{RBC}$, and plasma [14]. Thus, uptake of preformed 22:6n-3 from the diet may be critical for maintaining adequate membrane 22:6n-3 concentrations in vegetarians [14]. However, we did not collect socio-economic information for all subjects. Further studies are required to examine whether socio-economic information may modify the observed associations. In addition, our results showed a high composition of LC-PUFA compared to previous report. The methods used for fatty acids determination and population discrepancy may help explain this inconsistent result.

Previous studies and our present study demonstrated that n-3 PUFA and 22:6n-3 in plasma or red blood cell was significantly lower in vegetarians. Sanders et al. reported 
that the proportions of 22:6n-3 in plasma, blood cells, breast milk, and tissues are substantially lower in vegans and vegetarians compared with omnivores [15]. Kornsteiner et al. also demonstrates that vegetarians and vegans, who do not eat meat or fish, tend to have very low or negligible intakes of 20:5n-3 as well as 22:6n-3 [14]. Fokkema et al. investigated the PUFA status of Dutch vegans and omnivores in erythrocyte membranes. They found that 20:5n-3 and 22:6n-3 was significantly reduced in vegans compared with omnivores, while, a higher 22:5n-3 content compared with the Dutch omnivores [16], which is consistent with the present results. A study investigating plasma $n-3$ PUFA of British meat-eating, vegetarian, and vegan men showed that 20:5n-3, and 22:6n-3 were markedly decreased [17]. Li et al. demonstrated a decreased content of 20:5n-3, 22:6n-3 and total n-3 PUFA in the Australian vegetarian group [18]. Sanders et al. showed that the erythrocytes from vegans contained lower proportions of $20: 5 n-3,22: 5 n-3$ and 22:6n-3 and higher proportions of 18:2n-6, 20:2n-6 and 22:4n-6 [19]. Therefore, the low content of plasma PL n-3 PUFA reflects the limited n-3 PUFA dietary intake in vegetarians. The unbalanced $n-6: n-3$ ratio and the limited dietary sources of 20:5n-3 and 22:6n-3 in vegans and vegetarians led to reductions in 20:5n-3, 22:5n-3, 22:6n-3 and n-3 PUFA in PC, PS and PE compared with omnivores and semi-omnivores [14]. Vegetarian people are suggested to supplement fish oil or other food rich in n-3 PUFA to increase the level of $n-3$ PUFA in tissues $[14,20]$.

Inflammation plays a central role in development of atherosclerosis, CVD and type 2 diabetes [21]. In the present study, we found that vegetarians have higher IL6 concentrations. The possible mechanisms behind these associations are not fully understood. It was showed that elevated concentrations of pro-inflammatory cytokines such as IL-6 and acute phase reactants such as C-reactive protein are in turn independent risk factors for the development of type 2 diabetes [21] and CVD [22]. It has been shown that IL-6 may induce antagonists to inflammatory cytokines and therefore play a role in terminating inflammation [23]. The soluble TNF receptors, which are derived by proteolytic cleavage from TNF cell-surface receptors after induction by cytokines such as TNF, IL-6, IL-1 $\beta$, or IL-2 [24], bind to TNF and attenuate its bioactivity. Furthermore, we found LTB4, COX2 and PGE2 were lower in vegetarians. Interestingly, cell culture demonstrated that LTB4 synthesis can be modulated by the fatty acid composition of membrane phospholipids, which can be altered by dietary fatty acids [25]. Previous study reported that dietary supplements rich in n-3 PUFA reduce the concentrations of PGE2 and increase the synthesis of PGE3, which are believed to be less inflammatory. PGE2 and PGE3 both induce COX-2 mRNA via similar signaling mechanisms [26]. It was shown that increasing the $\mathrm{n}-3$ content of membrane phospholipid results in a decrease in mitogen-induced PGE2 synthesis.

This study demonstrated that replacement of $n-6$ PUFA with n-3 PUFA in cell membranes can result in a decreased cellular response to mitogenic and inflammatory stimuli [26].

Dietary factors substantially modulate inflammation and cardiovascular risk [27]. Long-chain n-3 PUFA reduces cardiovascular events and exerts well-established anti-inflammatory effects [28]. While, an increased ratio of dietary n-6 to n-3 PUFAs has been linked to the risk of chronic inflammatory diseases [28]. In the present study, we observed a light association between 22:6n-3 and IL-6. Interestingly, plasma 20:4n-6 was significantly positively correlated with TNF- $\alpha$ in Chinese male vegetarians. In consistent with our results, a cross-sectional study of dietary n-3 and n- 6 fatty acids and inflammatory markers also observed statistically significant inverse associations between $\mathrm{n}-3$ fatty acid intake and plasma levels of soluble TNF receptors 1 and 2 . The associations were restricted to the LC PUFA 20:5n-3 and 22:6n-3 and not 18:3n-3 [29]. At low levels of n-3 PUFA intake, n-6 PUFA are associated with high levels of inflammatory markers, yet at high levels of n-3 fatty acid intake, the combination of both types of fatty acids is related to the lowest levels of inflammation [29]. Despite a lower dietary PUFA intake, obese individuals have higher proportions of 20:4n-6 in their adipose tissue than do their non obese twins, which render their adipocytes more vulnerable to inflammation [30]. Human trials reported that n-3 fatty acid intake decreased inflammation. LC n-3 PUFA intakes were inversely associated with plasma concentrations of IL- 6 and matrix metalloproteinase-3. Non-fried fish consumption was found inversely related to C-reactive protein and IL-6; and fried fish was observed being inversely related to soluble intercellular adhesion molecules-1 (sICAM-1) but not associated with other biomarkers [31].

Intervention study showed that N-3 PUFA supplementation reduced plasma concentrations of soluble intercellular adhesion molecule-1, but had no significant effects on soluble vascular cell adhesion molecule-1, soluble P-selectin, or soluble E-selectin [32]. Treatment with LC n-3 PUFA favorably modulated adipose tissue and systemic inflammation in severely obese non-diabetic patients and improved lipid metabolism [27]. Perioperative fish oil infusions significantly increased PUFA concentrations in platelet and atrial tissue membranes within $12 \mathrm{~h}$ of the first FO administration and decreased biological and clinical signs of inflammation [33]. However, data from experimental studies of dietary n-3 PUFA and inflammatory markers reported quite conflicting results [34]. These experiments may be influenced by cell purification and culturing procedures and depend on cell origin 
and cell type, as TNF- $\alpha$ and IL- 6 are produced by a variety of cells.

One possible biological mechanism underlying the beneficial effects of $n-3$ PUFA on inflammation and endothelial function is that these fatty acids compete with $n-6$ fatty acids for prostaglandin and leukotriene synthesis at the cyclooxygenase and lipoxygenase level [31]. n-3 PUFA from fish or fish oil modulate prostaglandin metabolism by increasing prostaglandin $E_{3}$, thromboxane $A_{3}$ and leukotriene $B_{5}$ and by decreasing production of thromboxane $\mathrm{A}_{2}$ and leukotriene $\mathrm{B}_{4}$ formation [35]. Another suggested mechanism is that $n-3$ PUFA may react with active oxygen species because of their multiple double bonds and lead to a decreased production of hydrogen peroxide. Hydrogen peroxide is a critical activator of the nuclear factor- $\mathrm{kB}$ system of transcription factors that controls the coordinated expression of adhesion molecules and of leukocyte-specific chemoattractants upon cytokine stimulation [36]. Furthermore, the activities of the $\Delta 6$ and $\Delta 5$-desaturase and the activity of the cyclooxygenase are inhibited by both $n-3$ and n-6 PUFA [37]. Through this mechanism, high intake of PUFA could reduce inflammatory mediators through modulating inflammatory gene expression in immune cells [38]. PUFA may also modulate cytokine production or the release of the soluble TNF receptors through eicosanoid-independent pathways, for example, by influencing membrane composition and fluidity, affecting signal transduction processes or second messenger molecules, or binding to or affecting nuclear receptors such as the peroxisome proliferator receptors or nuclear factor-kB $[29,39]$.

In conclusion, vegetarians have higher IL- 6 and $n-6$ PUFA, but lower n-3 LC-PUFA, 22:6n-3, LTB4, COX2 and PGE2 levels. It would seem appropriate for vegetarians to increase their dietary 22:6n-3, while reduce dietary n-6 PUFA and thus reduce the risk of chronic inflammatoryrelated diseases.

\section{Competing interests}

There is no conflict of interests.

\section{Authors' contributions}

$X Y$ and $\mathrm{TH}$ carried out the experiment, participated in the study design and drafted the manuscript. XW, TS, and QW carried out the data analysis. XZ and $\mathrm{QH}$ participated in the design of the study and performed the statistical analysis. DL conceived of the study, and participated in its design and coordination. All authors read and approved the final manuscript.

\section{Acknowledgements}

This study was supported by a grant from Zhejiang Natural Science Foundation (Y2101133) and Zhejiang Science Foundation of Aging (2008ZJ003)

\section{Author details}

${ }^{1}$ Department of Clinical Laboratory, Zhejiang Hospital, 12 Linyin Road, Hangzhou 310030, China. ${ }^{2}$ Department of Nutrition, Harvard School of Public Health, Boston, USA. 'Department of Food Science and Nutrition, Zhejiang University, 866 Yuhangtang Road, Hangzhou 310059, China.
Received: 28 March 2014 Accepted: 14 September 2014 Published: 29 September 2014

\section{References}

1. Messina VK, Burke KI: Position of the American dietetic association: vegetarian diets. J Am Diet Assoc 1997, 97:1317-1321.

2. Huang T, Yang B, Zheng J, Li G, Wahlqvist ML, Li D: Cardiovascular disease mortality and cancer incidence in vegetarians: a meta-analysis and systematic review. Ann Nutr Metab 2012, 60:233-240.

3. Li D: Chemistry behind vegetarianism. J Agric Food Chem 2011, 59:777-784.

4. Davis BC, Kris-Etherton PM: Achieving optimal essential fatty acid status in vegetarians: current knowledge and practical implications. Am J Clin Nutr 2003, 78:640S-646S.

5. Janelle KC, Barr SI: Nutrient intakes and eating behavior scores of vegetarian and nonvegetarian women. J Am Diet Assoc 1995, 95:180-186. 189, quiz 187-188

6. Huang T, Yu X, Shou T, Wahlqvist ML, Li D: Associations of plasma phospholipid fatty acids with plasma homocysteine in Chinese vegetarians. Br J Nutr 2013, 109:1688-1694.

7. Lee HY, Woo J, Chen ZY, Leung SF, Peng XH: Serum fatty acid, lipid profile and dietary intake of Hong Kong Chinese omnivores and vegetarians. Eur J Clin Nutr 2000, 54:768-773.

8. Calder PC: n-3 polyunsaturated fatty acids, inflammation, and inflammatory diseases. Am J Clin Nutr 2006, 83:1505s-1519s.

9. James MJ, Gibson RA, Cleland LG: Dietary polyunsaturated fatty acids and inflammatory mediator production. Am J Clin Nutr 2000, 71:343s-348s

10. Endres S, Ghorbani R, Kelley VE, Georgilis K, Lonnemann G, van der Meer JW, Cannon JG, Rogers TS, Klempner MS, Weber PC, Schaefer EJ, Wolff SM, Dinarello CA: The effect of dietary supplementation with $n-3$ polyunsaturated fatty acids on the synthesis of interleukin- 1 and tumor necrosis factor by mononuclear cells. N Engl J Med 1989, 320:265-271.

11. Almario RU, Vonghavaravat $V$, Wong R, Kasim-Karakas SE: Effects of walnut consumption on plasma fatty acids and lipoproteins in combined hyperlipidemia. Am J Clin Nutr 2001, 74:72-79.

12. Li D, Sinclair A, Wilson A, Nakkote S, Kelly F, Abedin L, Mann N, Turner A: Effect of dietary alpha-linolenic acid on thrombotic risk factors in vegetarian men. Am J Clin Nutr 1999, 69:872-882.

13. Simopoulos AP: Essential fatty acids in health and chronic disease. Am J Clin Nutr 1999, 70:560S-569S.

14. Kornsteiner $M$, Singer I, Elmadfa I: Very low $n-3$ long-chain polyunsaturated fatty acid status in Austrian vegetarians and vegans. Ann Nutr Metab 2008, 52:37-47.

15. Sanders TA: DHA status of vegetarians. Prostaglandins Leukot Essent Fatty Acids 2009, 81:137-141.

16. Fokkema MR, Brouwer DA, Hasperhoven MB, Hettema Y, Bemelmans WJ, Muskiet FA: Polyunsaturated fatty acid status of Dutch vegans and omnivores. Prostaglandins Leukot Essent Fatty Acids 2000, 63:279-285.

17. Rosell MS, Lloyd-Wright Z, Appleby PN, Sanders TA, Allen NE, Key TJ: Long-chain $\mathrm{n}-3$ polyunsaturated fatty acids in plasma in British meat-eating, vegetarian, and vegan men. Am J Clin Nutr 2005, 82:327-334.

18. Li D, Ball M, Bartlett $M$, Sinclair A: Lipoprotein(a), essential fatty acid status and lipoprotein lipids in female Australian vegetarians. Clin Sci (Lond) 1999, 97:175-181.

19. Sanders TA, Ellis FR, Dickerson JW: Studies of vegans: the fatty acid composition of plasma choline phosphoglycerides, erythrocytes, adipose tissue, and breast milk, and some indicators of susceptibility to ischemic heart disease in vegans and omnivore controls. Am J Clin Nutr 1978, 31:805-813.

20. Huang T, Sinclair AJ, Shen LR, Yang B, Li D: Comparative effects of tuna oil and salmon oil on liver lipid metabolism and fatty acid concentrations in rats. J Food Lipids 2009, 16:436-451

21. Pradhan AD, Manson JE, Rifai N, Buring JE, Ridker PM: C-reactive protein interleukin 6, and risk of developing type 2 diabetes mellitus. JAMA 2001, 286:327-334.

22. Danesh J, Kaptoge S, Mann AG, Sarwar N, Wood A, Angleman SB, Wensley F, Higgins JP, Lennon L, Eiriksdottir G, Rumley A, Whincup PH, Lowe GD, Gudnason V: Long-term interleukin-6 levels and subsequent risk of coronary heart disease: two new prospective studies and a systematic review. PLoS Med 2008, 5:e78. 
23. Tilg H, Trehu E, Atkins MB, Dinarello CA, Mier JW: Interleukin-6 (IL-6) as an anti-inflammatory cytokine: induction of circulating IL-1 receptor antagonist and soluble tumor necrosis factor receptor p55. Blood 1994, 83:113-118.

24. Aderka D: The potential biological and clinical significance of the soluble tumor necrosis factor receptors. Cytokine Growth Factor Rev 1996, 7:231-240.

25. Dias VC, Wallace JL, Parsons HG: Modulation of cellular phospholipid fatty acids and leukotriene B4 synthesis in the human intestinal cell (CaCo-2). Gut 1992, 33:622-627.

26. Bagga D, Wang L, Farias-Eisner R, Glaspy JA, Reddy ST: Differential effects of prostaglandin derived from omega- 6 and omega-3 polyunsaturated fatty acids on COX-2 expression and IL-6 secretion. Proc Natl Acad Sci U S A 2003, 100:1751-1756.

27. Itariu BK, Zeyda M, Hochbrugger EE, Neuhofer A, Prager G, Schindler K, Bohdjalian A, Mascher D, Vangala S, Schranz M, Krebs M, Bischof MG, Stulnig TM: Long-chain n-3 PUFAs reduce adipose tissue and systemic inflammation in severely obese nondiabetic patients: a randomized controlled trial. Am J Clin Nutr 2012, 96:1137-1149.

28. Calder PC: Polyunsaturated fatty acids and inflammatory processes: New twists in an old tale. Biochimie 2009, 91:791-795.

29. Pischon T, Hankinson SE, Hotamisligil GS, Rifai N, Willett WC, Rimm EB: Habitual dietary intake of $n-3$ and $n-6$ fatty acids in relation to inflammatory markers among US men and women. Circulation 2003, 108:155-160.

30. Pietilainen KH, Rog T, Seppanen-Laakso T, Virtue S, Gopalacharyulu P, Tang J, Rodriguez-Cuenca S, Maciejewski A, Naukkarinen J, Ruskeepaa AL, Niemelä PS, Yetukuri L, Tan CY, Velagapudi V, Castillo S, Nygren H, Hyötyläinen T, Rissanen A, Kaprio J, Yki-Järvinen H, Vattulainen I, Vidal-Puig A, Orešič M: Association of lipidome remodeling in the adipocyte membrane with acquired obesity in humans. PLOS Biol 2011, 9:e1000623.

31. He K, Liu K, Daviglus ML, Jenny NS, Mayer-Davis E, Jiang R, Steffen L, Siscovick D, Tsai M, Herrington D: Associations of dietary long-chain $\mathrm{n}-3$ polyunsaturated fatty acids and fish with biomarkers of inflammation and endothelial activation (from the Multi-Ethnic Study of Atherosclerosis [MESA]). Am J Cardio/ 2009, 103:1238-1243.

32. Yang $Y, L u N$, Chen D, Meng L, Zheng $Y$, Hui R: Effects of $n-3$ PUFA supplementation on plasma soluble adhesion molecules: a meta-analysis of randomized controlled trials. Am J Clin Nutr 2012, 95:972-980.

33. Berger MM, Delodder F, Liaudet L, Tozzi P, Schlaepfer J, Chiolero RL, Tappy L: Three short perioperative infusions of $n-3$ PUFAs reduce systemic inflammation induced by cardiopulmonary bypass surgery: a randomized controlled trial. Am J Clin Nutr 2013, 97:246-254.

34. Blok WL, Katan MB, van der Meer JW: Modulation of inflammation and cytokine production by dietary (n-3) fatty acids. J Nutr 1996, 126:1515-1533.

35. Leaf A, Weber PC: Cardiovascular effects of $n-3$ fatty acids. N Engl J Med 1988, 318:549-557.

36. De Caterina R, Zampolli A: n-3 fatty acids: antiatherosclerotic effects. Lipids 2001, 36(Suppl):S69-78.

37. Cho HP, Nakamura MT, Clarke SD: Cloning, expression, and nutritional regulation of the mammalian Delta-6 desaturase. J Biol Chem 1999, 274:471-477.

38. Zeyda M, Stulnig TM: Lipid Rafts \& Co.: an integrated model of membrane organization in T cell activation. Progr Lipid Res 2006, 45:187-202.

39. Graber R, Sumida C, Nunez EA: Fatty acids and cell signal transduction. J Lipid Mediat Cell Signal 1994, 9:91-116.

doi:10.1186/1476-511X-13-151

Cite this article as: Yu et al:: Plasma n-3 and n-6 fatty acids and inflammatory markers in Chinese vegetarians. Lipids in Health and Disease 2014 13:151.

\section{Submit your next manuscript to BioMed Central and take full advantage of:}

- Convenient online submission

- Thorough peer review

- No space constraints or color figure charges

- Immediate publication on acceptance

- Inclusion in PubMed, CAS, Scopus and Google Scholar

- Research which is freely available for redistribution

Submit your manuscript at www.biomedcentral.com/submit
C Biomed Central 